\title{
artigo
}

\section{DimeRede: desenvolvimento de uma proposta de metodologia para o planejamento e dimensionamento da força de trabalho em saúde}

\author{
DimeRede: development of a methodology for health workforce planning and sizing
}

DimeRede: desarrollo de una metodología para el dimensionamiento del personal sanitario

\begin{abstract}
RESUMO
Objetivo: Descrever o desenvolvimento de uma metodologia para o planejamento e dimensionamento da força de trabaIho multiprofissional em saúde, integrando os três níveis da Rede de Atenção à Saúde (RAS) do Sistema Único de Saúde (SUS); Método: relato de experiência da construção dessa proposta metodológica, realizada entre abril de 2019 e março de 2020, fundamentada nas diretrizes do SUS, na literatura e nas vivências nos projetos do Ministério da Saúde; Resultado: apresentação de uma metodologia, denominada DimeRede que, dirigida ao arranjo organizativo das RAS, estima a força de trabalho necessária de acordo com as necessidades locorregionais, considerando o perfil epidemiológico e os determinantes sociais de saúde e adoecimento identificados em cada estratificação territorial; Conclusão: a DimeRede constitui um avanço importante, mostrando-se potente para instrumentalizar políticas de gestão do trabalho. Contudo, sem encerrar os desafios de garantir equipes adequadas para o cuidado multiprofissional conforme as necessidades de saúde da população.
\end{abstract}

DESCRITORES: Recursos humanos; Gestão em saúde; Regionalização; Dimensionamento de pessoal.

\section{ABSTRACT}

Goal: To describe the development of a methodology for planning and sizing of the multi-professional health workforce, by integrating the three levels of the Health Care Network (RAS) of the Unified Health System (SUS). Methodology: experience report on the elaboration of this methodological proposal, held between April 2019 and March 2020, based on SUS guidelines, literature and experiences in projects of the Ministry of Health. Results: presentation of DimeRede, which, oriented to the organizational arrangement of the RAS, estimates the necessary workforce according to the local and regional needs, taking into consideration the epidemiological profile and social determinants of health and illness identified in each territorial stratification. Conclusion: the DimeRede approach constitutes an important advance, proving to be a powerful instrument to implement labor management policies. However, it doesn't end the challenges of ensuring adequate teams for multi-professional care in accordance with the health needs of the population.

DESCRIPTORS: Workforce; Health management; Regional health planning; Personnel downsizing.

\section{RESUMEN}

Objetivo: Describir el desarrollo de una metodología para la planificación y dimensionamiento del personal sanitario multiprofesional, integrando los tres niveles de la Red de Atención Médica (RAS) del Sistema Unificado de Salud (SUS); Método: informe de la experiencia de la construcción de esta propuesta metodológica, realizada entre abril de 2019 y marzo de 2020, basado en las directrices, literatura y experiencias del SUS en los proyectos del Ministerio de Salud; Resultado: presentación de una metodología, llamada DimeRede, que, dirigida a la disposición organizativa del RAS, estima la fuerza de trabajo necesaria de acuerdo con las necesidades regionales locales, considerando el perfil epidemiológico y los determinantes sociales de salud y enfermedad identificados en cada estratificación territorial; Conclusión: DimeRede es un avance importante, demostrando ser poderoso para instrumentalizar las políticas de gestión del trabajo. Sin embargo, sin cerrar los retos de garantizar equipos adecuados para la atención multiprofesional de acuerdo con las necesidades de salud de la población.

DESCRIPTORES: Recursos humanos; Gestión de la salud; Regionalización; Reducción de personal.

RECEBIDO EM: 29/11/2020 APROVADO EM: 08/12/2020 


\section{Desirée dos Santos Carvalho}

Enfermeira e professora, especialista de Gestão do Trabalho e da Educação na Saúde e mestra em Saúde Coletiva. Trabalhadora do SUS no Grupo Hospitalar Conceição/Ministério da Saúde, foi tutora no Projeto de Governança da Gestão do Trabalho em Saúde como pesquisadora no Instituto Brasileiro de Informação em Ciência e Tecnologia/Ministério da Ciência Tecnologia e Inovação, atualmente é pesquisadora do Centro de Centro de Inovação em Gestão da Educação e do Trabalho em Saúde CIGETS na Universidade Federal de Goiás.

ORCID: 0000-0002-9223-6891

\section{Elisabet Pereira Lelo Nascimento}

Enfermeira sanitarista, mestra em Enfermagem e doutora em Saúde Coletiva. Apoio técnico do Departamento de Gestão do Trabalho e Educação na Saúde da Secretaria Municipal de Saúde de Campinas, foi coordenadora do Projeto de Governança da Gestão do Trabalho em Saúde como pesquisadora no Instituto Brasileiro de Informação em Ciência e Tecnologia/Ministério da Ciência Tecnologia e Inovação, atualmente é pesquisadora do Centro de Centro de Inovação em Gestão da Educação e do Trabalho em Saúde - CIGETS na Universidade Federal de Goiás.

ORCID: 0000-0001-8649-6649

\section{INTRODUÇÃO}

$\mathbf{P}$ ara a sustentabilidade do Sistema Único de Saúde (SUS), na perspectiva de uma atenção universal e integral, faz-se necessário superar a fragmentação sistêmica e o modelo estruturado em ações e serviços de saúde que são planejados exclusivamente a partir da oferta já existente. Segundo o Ministério da Saúde (MS), a organização do sistema em Redes de Atenção à Saúde (RAS) é a principal estratégia para a mudança do sistema de saúde tanto do ponto de vista da organização administrativa e financeira, quanto da qualificação do cuidado prestado à população ${ }^{1}$.

Os países abordam comumente o planejamento da força de trabalho em saúde a partir do perfil dos serviços ofertados, resultando no dimensionamento de equipes padrão2. Estes modelos culminam em uma gestão do trabalho ineficiente por não considerarem questões externas às organizações, como a diversidade dos territórios e das necessidades de saúde dos usuários ${ }^{2-3}$. Logo, fatores como o envelhecimento da população e as características de morbimortalidade da população tornam imprescindível um dimensionamento da força de trabalho em saúde eficiente, eficaz e orientado para todos os níveis de atenção².

No Brasil são utilizados diversos modos de dimensionamento das necessidades de força de trabalho em saúde. Alguns deles, em especial as experiências incenti- vadas ou divulgadas pelo MS, sinalizam avanços ao considerar mais de uma categoria profissional e despertar para a lógica das linhas de cuidado e da organização em rede $^{4}$. Por outro lado, a produção científica evidencia que no país há uma tendência de planejamento da força de trabalho por meio de métodos normativos e com maior frequência dirigidos especificamente para as equipes de enfermagem e hospitalar ${ }^{2,5}$.

Modificar essa tendência exige um olhar amplo para as regiões e redes de saúde, constituindo desafio à conformação da RAS com modelo de atenção centrado nas necessidades de saúde da população. Portanto, é relevante ampliar estudos e compartilhar modelos e práticas de dimensionamento da força de trabalho necessária para o alcance da atenção integral em saúde ${ }^{6}$.

O desenvolvimento de metodologias de dimensionamento da força de trabalho é um desafio enfrentado pela Secretaria de Gestão do Trabalho e da Educação na Saúde (SGTES) do MS nos últimos anos. Neste sentido, no ano de 2019 demandou que um grupo de especialistas construísse uma metodologia que envolvesse mais de um serviço ou nível de atenção.

O objetivo deste artigo é descrever essa experiência de desenvolvimento de uma metodologia para o planejamento e dimensionamento da força de trabalho multiprofissional em saúde, a partir da integração dos três níveis da Rede de Atenção à Saúde do Sistema Único de Saúde.

\section{METODOLOGIA}

Trata-se de um relato de experiência vivida pelas autoras, enquanto trabalhadoras do SUS e pesquisadoras da área de gestão do trabalho, no desenvolvimento de uma proposta de metodologia para o planejamento e dimensionamento da força de trabalho em saúde, realizada por demanda do Departamento de Gestão do Trabalho em Saúde (DEGTS) da SGTES/MS

Os atores envolvidos no processo foram técnicos e gestores do DEGTS e a equipe implicada nas atividades do Projeto de Governança da Gestão do Trabalho, desenvolvido à época com objetivo de implementar atividades de dimensionamento na primeira região de saúde no estado do Ceará. Neste contexto, a primeira autora atuou como responsável pela construção da proposta sob coordenação da segunda, e os demais contribuíram para a discussão, as reflexões e a validação da metodologia.

O estudo metodológico foi realizado entre abril de 2019 e março de 2020 e compreendeu quatro fases: o delineamento dos objetivos; a definição dos pressupostos; a elaboração da matriz; e a validação.

O delineamento dos objetivos foi realizado a partir de reuniões com os gestores do DEGTS. A solicitação foi que a metodologia a ser construída pudesse unir características de metodologias já utilizadas pelo MS e ser aplicada em mais de um tipo de serviço. Também que pudesse gerar, a partir de dados secundários, 


\section{artigo}

Carvalho, D.S.; Nascimento, E.P.L.;

DimeRede: desenvolvimento de uma proposta de metodologia para o planejamento e dimensionamento da força de trabalho em saúde

subsídios para as atividades da SGTES e de outras secretarias do MS no apoio aos gestores municipais e estaduais, bem como na formulação de políticas públicas de gestão do trabalho.

Desta forma, destaca-se que a construção da metodologia não partiu do ponto zero e restrito. A necessidade de um método mais abrangente já fazia parte das discussões desse e de outros grupos de profissionais e gestores que aplicam e demandam metodologias de dimensionamento no cotidiano das organizações de saúde. Por isso, inspirou-se nas experiências descritas na literatura e nas vivências produzidas em projetos anteriores, financiados pela SGTES, tais como o Dimensionamento da Atenção Básica e o Planejamento e Dimensionamento da Força de Trabalho da Rede de Urgência e Emergência e do próprio projeto de Governança da Gestão do Trabalho em Saúde ${ }^{7-9}$. Através da revisão não sistemática da literatura, das normativas do SUS e dos documentos produzidos nessas experiências, além de reuniões e rodas de conversa com os atores envolvidos, os pressupostos foram definidos: arranjo organizativo das RAS; atenção integral e regionalizada; cuidado multiprofissional; modelo de atenção centrado nas necessidades da população.

A elaboração da matriz da metodologia buscou conjugar elementos presentes nas experiências e projetos citados: ques- tões norteadoras e análise da rede e do território como etapa anterior ao cálculo de dimensionamento ${ }^{7-9}$. Para tanto, foram delineadas as questóes norteadoras consideradas essenciais para o desenvolvimento do método conforme os objetivos e pressupostos, e organizadas em uma matriz composta por quatro etapas.

Ao longo do período foram realizadas diversas apresentações da proposta de metodologia para distintos grupos implicados com essa temática. Considerando as discussões nestes espaços, as diretrizes do SUS e a fundamentação teórica, foram pactuadas as direções e revisadas exaustivamente as possibilidades e especificidades da demanda junto aos gestores do DEGTS. A validação dos pressupostos e da matriz da metodologia se deu por meio dessas apresentações aos atores envolvidos.

Em outubro de 2019, o DEGTS aprovou os pressupostos e as etapas e autorizou o seguimento e a organização de projeto-piloto para construir uma ferramenta de tecnologia de informação que viabilizasse a aplicação da metodologia. Posteriormente, produziu-se um documento com a descrição detalhada da metodologia proposta e procedeu-se com a apresentação para técnicos de outras secretarias do MS, relacionadas à gestão da atenção à saúde, para qualificar a discussão sobre as variáveis a serem utilizadas.

\section{RESULTADOS E DISCUSSÃO}

Desenvolveu-se uma metodologia que pretende gerar uma estimativa rápida e consistente da força de trabalho necessária para a composição da RAS, considerando todas as categorias profissionais envolvidas e integrando os três níveis de atenção. De acordo com os pressupostos, o processo de construção da metodologia amparou-se em referências de normativas sobre redes do SUS. À vista disso, convencionou-se denominá-la de DimeRede.

As quatro etapas da metodologia proposta, nominadas no Quadro 1, são complementares entre si e visam, em conjunto, planejar e dimensionar a força de trabalho em saúde compreendendo a organização da RAS a partir das necessidades de saúde da população e das características do território.

O Diagnóstico Situacional tem como objetivo amparar o planejamento da força de trabalho em saúde, organizando os dados encontrados em bases de dados nacionais para identificar as características de cada município, as condições de vida e saúde das pessoas vivendo no território, os estabelecimentos, a produção e a força de trabalho disponíveis nos três níveis de atenção. Sendo assim, leva em consideração o pressuposto de que a assistência integral se inicia e se completa na RAS e o planejamento da saúde deve ser realiza-

Quadro 1. Etapas e principais questões norteadoras da metodologia DimeRede.

$$
\text { DIAGNÓSTICO SITUACIONAL }
$$

Quais são as características desse território? A qual região de saúde ele pertence?

Como vivem, adoecem e morrem os usuários integrantes dessa população?

Quais estabelecimentos de saúde estão disponiveis? Quais serviços ofertam? Com qual força de trabalho contam atualmente?

\section{ESTIMATIVA DE AÇÕES E SERVIÇOS PÚBLICOS DE SAÚDE}

Quais são os serviços e ações de saúde que devem ser ofertados neste território?

Onde eles devem estar distribuídos conforme a regionalização estabelecida e políticas públicas vigentes?

Qual a quantidade estimada de acordo com as necessidades de saúde da população?

\section{DIMENSIONAMENTO DA FORÇA DE TRABALHO}

Quais as categorias profissionais que devem compor as equipes de saúde que ofertarão os serviços estimados para essa RAS? Quantas horas de trabalho são necessárias para atender as necessidades de saúde dessa população?

Quantos trabalhadores de saúde esse conjunto de horas representam?

\section{ANÁLISE COMPARATIVA}

Comparando os resultados do diagnóstico situacional com as estimativas de serviços, ações e força de trabalho necessárias para compor a RAS, há suficiência? Em quais territórios temos insuficiências? A força de trabalho está distribuída adequadamente?

Fonte: Elaborado pelas autoras. 
do de maneira regionalizada, a partir das necessidades dos municípios ${ }^{10}$. Por essa razão, utiliza exclusivamente as informações e os indicadores com dados disponíveis em agregado municipal, tais como: número de habitantes; estimativa de população por faixa etária e sexo; PIB per capita; percentual da população em extrema pobreza; população em situação de rua; percentual da população com plano de saúde; esgotamento sanitário; internações por condições sensíveis à atenção básica; cobertura de Estratégia Saúde da Família; número de estabelecimentos por nível de atenção; número de profissionais cadastrados, entre outros.

A escolha por dados secundários, além de viabilizar maior validade para a comparação entre os territórios estudados, garante as mesmas fontes e possibilita economia de tempo e recursos que seriam necessários para coleta de dados primários. Ao mesmo tempo, tem envolvimento indireto dos usuários (respondedores dos inquéritos populacionais) e dos trabalhadores e gestores (responsáveis pela produção dos dados no sistema de saúde). Assim como aproveita o potencial de uso das informações sistematizadas como as da Fundação Instituto Brasileiro de Geografia e Estatística (IBGE) e do Departamento de Informática do SUS (DataSUS) para o planejamento em saúde.

A organização da RAS exige a definição da Região de Saúde, bem como de seus limites geográficos, a população e o estabelecimento do rol de ações e serviços que nela serão ofertados ${ }^{1}$. Consequentemente, além da organização no espaço geográfico e federativo (município e unidade da Federação), o método considera estas definições de Regiões e Macrorregiões de Saúde, estabelecidas em pactuações tripartites e cujos registros estejam disponíveis nos bancos de dados nacionais, para organizar as sínteses de informações conforme as escalas de abrangência da RAS e classificar os territórios.

A necessidade de diferenciar os territórios conforme as condições de cada um, para além da regionalização em saúde foi bastante discutida, sendo consenso que

A necessidade

de diferenciar os

territórios conforme

as condições de cada

um, para além da

regionalização em

saúde foi bastante

discutida, sendo

consenso que

espaços geográficos

de uma mesma

região de saúde

podem apresentar

discrepância de

outros fatores que

influenciam na

capacidade do

setor saúde. espaços geográficos de uma mesma região de saúde podem apresentar discrepância de outros fatores que influenciam na capacidade do setor saúde. Pesquisaram-se formas de estratificação por meio de revisão não sistemática da literatura e da busca por relatos orais e escritos de experiências e definição das regiões de saúde nos estados brasileiros.

A ferramenta que atendeu as expectativas foi a publicada por pesquisadores que, ao reconhecer que os territórios se encontram em diferentes estágios de organização e desenvolvimento devido suas diversidades históricas, culturais e ambientais, desenvolveram, validaram um método que estratifica os municípios brasileiros em grupos homogêneos por meio do porte populacional e de um conjunto de indicadores que, correlacionados, influenciam nas condições de gestão em saúde. Estratifica os municípios em três portes populacionais (grande, médio e pequeno) e, ao considerar os resultados das somas de indicadores que apontam as características demográficas (densidade demográfica e taxa de urbanização), o poder aquisitivo da população (percentual em extrema pobreza e cobertura de plano de saúde) e a capacidade de financiamento do município (PIB per capita), classifica os de pequeno e médio porte em condiçôes favoráveis ou desfavoráveis para a gestão ${ }^{11}$.

Optou-se pela utilização dessa forma validada de estratificação dos municípios de acordo com as condições para a gestão, somadas as classificações relacionadas à abrangência dos serviços de saúde e a uma subdivisão para os municípios de grande porte. Portanto, os territórios são estratificados em onze tipos na DimeRede, conforme detalhado no Quadro 2. Atenta-se para o fato de que o território sanitário no qual se organiza a RAS exige demarcar o conjunto de ações e serviços que garantirão acessibilidade e sustentabilidade operacional, além das definições geográficas e populacionais ${ }^{1}$.

As sínteses produzidas nesta etapa são utilizadas nas classificações, nos cálculos e nas comparações das etapas seguintes.

O planejamento e organização das 


\section{artigo}

Carvalho, D.S.; Nascimento, E.P.L.;

DimeRede: desenvolvimento de uma proposta de metodologia para o planejamento e dimensionamento da força de trabalho em saúde

Ações e Serviços Públicos de Saúde (ASPS) que integram uma rede regionalizada e hierarquizada no SUS devem acontecer de forma ascendente, considerando a diversidade no processo de implementação das RAS existentes no país, o interesse regional, as responsabilidades dos entes para com essas ações e serviços, os planos de saúde aprovados pelos conselhos de saúde e as decisões pactuadas nas Comissões Intergestores Bipartites $^{12}$. Reconhecendo que esse complexo planejamento das ASPS não pode ser realizado ou apurado de forma centralizada com dados secundários, esta etapa limita-se a estimar o conjunto de ações e serviços que seriam pactuados nos territórios caso todas as diretrizes e políticas nacionais comuns fossem levadas em consideração.

Denominamos este conjunto de procedimentos e intervenções de Estimativa de Ações e Serviços Públicos de Saúde (EASPS), na qual propóe-se que cada classificação territorial corresponda a um agrupamento de ações e serviços necessários, levando em conta a perspectiva de que a modelagem das RAS é realizada a partir da análise da situação de saúde dos territórios e tem como fundamento a suficiência, proposta numa matriz por nível de atenção (Quadro 2) ${ }^{1,12}$. Posteriormente, verificam-se as ações necessárias para os conjuntos descritos acima e dimensiona-se a quantidade necessária para cada território tendo em vista as diretrizes clínicas e a população-alvo, conforme parâmetros SUS, de acordo com os perfis demográficos e epidemiológicos identificados no Diagnóstico Situacional.

A definição e disponibilização de parâmetros nacionais e regionalizados para orientar o planejamento e a programação das ações e dos serviços de saúde, o compromisso com a implementação de modelo de atenção à saúde que atenda às políticas pactuadas e às necessidades de saúde da população brasileira são diretrizes para os processos de Regionalização, Planejamento Regional Integrado e Go- vernança das Redes de Atenção à Saúde que devem ser observadas por todos os gestores do SUS ${ }^{1,10,13}$. Portanto, esta etapa depende da pesquisa, da interpretação e da sistematização dos documentos orientadores do Ministério da Saúde e das Comissões Intergestores Tripartite e Bipartite, além das regulamentações e dos princípios do SUS, para aportar os indicadores e parâmetros a compor cada conjunto. Este processo, assim como as necessidades de saúde, é dinâmico e, portanto, suas variáveis devem ser constantemente revisadas e periodicamente atualizadas.

O Dimensionamento da força de trabalho em saúde é a etapa que se refere ao cálculo do número de trabalhadores necessários em si. É o momento em que são aplicados os parâmetros, conforme os dados e resultados das etapas anteriores, a depender da composição e escopo de práticas planejadas para a equipe e para cada categoria profissional que a comporá ${ }^{14}$. Cabe destacar que esse processo é comple-

Quadro 2. Representação da estratificação dos territórios sanitários e estimativa de ações e serviços públicos de saúde (EASPS) conforme as diretrizes de organização da RAS.

\begin{tabular}{|c|c|c|}
\hline ESTRATIFICAÇÃO & EASPS & \\
\hline Unidade da Federação & Conjunto A & \multirow{2}{*}{ Atenção Terciária } \\
\hline Macrorregião de Saúde & Conjunto B & \\
\hline Região de Saúde & Conjunto C & Atenção Secundária \\
\hline $\begin{array}{l}\text { Município de Grande } \\
\text { Acima de } 500 \text { mil habitantes }\end{array}$ & Conjunto D & \multirow{8}{*}{ Atenção Primária } \\
\hline $\begin{array}{l}\text { Município de Grande <500 mil } \\
\text { Acima de } 100 \text { mil habitantes }\end{array}$ & Conjunto $\mathrm{E}$ & \\
\hline $\begin{array}{l}\text { Município de Médio Favorável } \\
25 \text { a } 100 \text { mil habitantes e influentes favoráveis para gestão }\end{array}$ & Conjunto F & \\
\hline $\begin{array}{c}\text { Município de Médio Regular } \\
25 \text { a } 100 \text { mil habitantes e influentes regulares para gestão }\end{array}$ & Conjunto G & \\
\hline $\begin{array}{c}\text { Município de Médio Desfavorável } \\
25 \text { a } 100 \text { mil habitantes e influentes desfavoráveis para gestão }\end{array}$ & Conjunto $\mathrm{H}$ & \\
\hline $\begin{array}{c}\text { Município de Pequeno Favorável } \\
\text { Até } 25 \text { mil habitantes e influentes favoráveis para gestão }\end{array}$ & Conjunto I & \\
\hline $\begin{array}{c}\text { Município de Pequeno Regular } \\
\text { Até } 25 \text { mil habitantes e influentes regulares para gestão }\end{array}$ & Conjunto J & \\
\hline $\begin{array}{c}\text { Município de Pequeno Desfavorável } \\
\text { Até } 25 \text { mil habitantes e influentes desfavoráveis para gestão }\end{array}$ & Conjunto K & \\
\hline
\end{tabular}


xo, especialmente diante da premência de articular as necessidades de diferentes esferas públicas que compartilham responsabilidades, e requer uma visão ampliada sobre saúde ${ }^{6}$.

A fragmentação dos serviços e de práticas de saúde é expressa também pela fragilidade da gestão do trabalho, pela precarização dos vínculos e pela quantidade insuficiente de profissionais. Por isso, a busca de soluções para os problemas de saúde da população exige equipes multiprofissionais, valorização do trabalho, além de práticas compartilhadas de clínica ampliada ${ }^{1}$. Dessa forma, a DimeRede considera todas as categorias profissionais que compõem a equipe e a partir da EASPS, calculando quantas horas de trabalho seriam necessárias para atender a população de cada estrato territorial. Este resultado em horas é transformado em profissionais equivalentes conforme uma carga horária padrão $0^{8,14}$.

Para ilustrar e facilitar a compreensão do leitor, no Quadro 3 usamos como exemplo o dimensionamento da força de trabalho para uma Região de Saúde fictícia, considerando especificamente consultas do componente pré-natal da Rede Cegonha, uma das redes temáticas da RAS1. Levando em conta que a região teve 1905 nascidos vivos no ano anterior, a partir dos parâmetros assistenciais SUS, estima-se 2000 gestantes e, portanto, a necessidade de ações e serviços nas quantidades descritas no quadro ${ }^{15}$.

Ressaltamos que este exemplo de dimensionamento corresponde a um fragmento do conjunto de EASPS previsto para esta região e que os profissionais realizam diversas intervenções em saúde, não apenas atendimentos individuais, que seriam somadas a essas horas necessárias de cada categoria profissional a fim de compor o número de profissionais equivalente para a atenção integral aos usuários.

No caso apresentado, consideraram-se consultas de 30 minutos, porém, não há definição exata desse tempo nos materiais do Ministério da Saúde. Assim como há previsão de consultas puerperais e psicossociais, podendo ser realizadas por mais de uma categoria. No entanto, não está prevista uma divisão estática entre as profissões para o trabalho compartilhado ${ }^{15}$.

$\mathrm{Na}$ construção das duas últimas etapas descritas, destacaram-se as lacunas referentes aos padrões mínimos sugeridos para determinados serviços, bem como a ausência ou, em alguns casos, as múltiplas orientações de parâmetros de força de trabalho e de oferta recomendados para algumas ações cujas políticas nacionais estão publicadas. Assim, aponta a necessidade de buscar consensos nas comissões intergestores e instâncias participativas do SUS, para eleger as variáveis que melhor se apliquem a esta metodologia, considerando as melhores evidências e o modelo de atenção à saúde.

A Análise Comparativa é a etapa final, em que confronta os resultados encontrados no Diagnóstico Situacional com os resultados das etapas de EASPS e Dimensionamento da Força de Trabalho, verificando se há adequação ou diferenças entre eles, produzindo sínteses e análises confor- me os níveis de atenção e as estratificações dos territórios.

Com isso a DimeRede identifica os territórios, as necessidades locorregionais, os estabelecimentos de saúde e a força de trabalho existentes por meio de dados secundários. Ela planeja e dimensiona a força de trabalho multiprofissional necessária a partir da EASPS. Agrega ao planejamento normativo uma abordagem estratégica à medida que propõe dimensionar a atenção que deve ser prestada, conforme as políticas de saúde vigentes, e não a que já é ofertada. É capaz de produzir informações e análises que podem colaborar para formulação de políticas públicas de gestão do trabalho e a tomada de decisão em dimensões sistêmicas, sendo indicada para situações que exigem o estabelecimento de referências comuns a todos ou para uso combinado de outras metodologias conforme os objetivos. O próximo passo é a definição e validação das variáveis, construção de viabilidade em termos de tecnologia da informação e a aplicação em projeto-piloto.

\section{CONCLUSÃO}

A proposição da metodologia DimeRede constitui um avanço na perspectiva da identificação das necessidades da força de trabalho necessária de acordo com o modelo de saúde que se pretende fortalecer e consolidar no SUS, superando a lógica da reprodução e manutenção de práticas com foco uniprofissional e/ ou que observam serviços de saúde de forma isolada ou a partir das ofertas já

Quadro 3. Simulação do dimensionamento da força de trabalho considerando parte do Conjunto EASPS definido à uma Região de Saúde fictícia.

\begin{tabular}{|c|c|c|c|}
\hline $\begin{array}{c}\text { AÇÕES E SERVIÇOS } \\
\text { NECESSÁRIOS (QUANTIDADE) }\end{array}$ & PROFISSIONAL & $\begin{array}{c}\text { NÚMERO HORAS DE TRABALHO } \\
\text { NECESSÁRIAS NO ANO }\end{array}$ & $\begin{array}{c}\text { NÚMERO DE PROFISSIONAIS } \\
\text { EQUIVALENTES(130H/MÊS) }\end{array}$ \\
\hline Consultas odontológicas (2000) & Dentista & 1000 & 0,64 \\
\hline Consultas pré-natal (6000) & Médica & 3000 & 1,92 \\
\hline Consultas de enfermagem (6000) & Enfermeira & 3000 & 1,92 \\
\hline Consultas obstétricas (1500) & $\begin{array}{c}\text { Médica Ginecologista e } \\
\text { Obstetra }\end{array}$ & 750 & 0,48 \\
\hline Fonte: Elaborado pelas autoras. & \multicolumn{3}{|l}{} \\
\hline
\end{tabular}




\section{artigo}

realizadas. É potente para instrumentalizar políticas de gestão do trabalho como a prospecção de postos de trabalho, a identificação de necessidades de formação, a distribuição de vagas de residências médica e multiprofissional, a implantação de planos de carreiras e de incentivos para provimento e fixação de profissionais, consoante às necessidades de cada região de saúde.

As aprendizagens e reflexões suscitadas no processo de construção do método asseguram a relevância e complexidade dessa abordagem. Deste modo, cabe destacar que a elaboração de uma metodologia de planejamento e de dimensionamento da força de trabalho em saúde pautada na conformação da Rede de Atenção à Saúde não encerra os desafios de garantir equipes adequadas para o cuidado multiprofissional segundo as necessidades de saúde da população de um determinado território visto que, desde já, em seu modelo teórico, desvela lacunas referentes a falta de clareza de diretrizes sobre parâmetros para algumas ações e serviços públicos de saúde e para determinadas práticas e categorias profissionais. Ainda que as definições dessas importantes variáveis envolvam outras áreas da gestão do SUS, perpassando acordos intergestores e regulações das profissões, o sucesso prático da DimeRede depende também da resolução dessas lacunas. Entende-se, então, que é imprescindível pesquisar e pactuar parâmetros de atenção e de força de trabalho, aliando as experiências práticas e as diretrizes do SUS com critérios científicos, bem como desenvolver ferramentas de tecnologias da informação que apoiem a sistematização dos dados de cada território e a aplicação da metodologia. -

\section{REFERÊNCIAS}

1. Brasil. Ministério da Saúde. Portaria de Consolidação n 3, de 28 de setembro de 2017 . Consolidação das normas sobre as redes do Sistema Único de Saúde. Diário Oficial da União. 03 out. 2017.

2. Machado CR, Dal Poz MR. Sistematização do conhecimento sobre as metodologias empregadas para o dimensionamento da força de trabalho em saúde. Saúde debate [Internet]. 2015 mar. [acesso em 2020 ago. 10]; 39(104): 239-54. Disponivel em: http://www.scielo.br/scielo.php?script=sci_arttext\&pid=S0103-11042015000100239\&Ing=en.

3. Vianna, CMM, Pierantoni CR, França TC, et al. Modelos econométricos de estimativa da força de trabalho: uma revisão integrativa da literatura. Physis [Internet]. 2013 set [acesso em 2020 nov. 12]; 23(3):925-50. Disponível em: $\quad$ http://www.scielo.br/scielo.php?script=sci_arttext\&pid=S0103-73312013000300014\&Ing=en.

4. Ramos LB, Possa LB. Dimensionamento da força de trabalho no SUS: o trabalho (e trabalhador) vivo no planejamento do cuidado em saúde. Saúde em redes [Internet]. 2016. [acesso em 2020 ago 12]; 2(1):43-52. Disponivel em: http://revista.redeunida.org.br/ojs/index.php/rede-unida/article/view/653/pdf_10

5. Moura, LP. Planejamento da força de trabalho em saúde: uma análise do período de 1964 a 2013. [Dissertação]. Salvador: Instituto de Saúde Coletiva da UFBA; 2014.

6. Carvalho DS, Nascimento EPL, Souza GH. Desafios do dimensionamento da força de trabalho para a conformação da rede de atenção à saúde. SaudColetiv (Barueri). No prelo 2020.

7. Nascimento EPL, Valle MLF, Carmona SAMLD, Barthmann VMC, organizadoras. Dimensionamento da força de trabalho da atenção básica: Sistema Único de Saúde. Campinas, 2019.

8. Possa LB.; Trepte RF, organizadoras. Planejamento e dimensionamento da força de trabalho para os serviços pré-hospitalares fixos e hospitalares de urgência e emergência: desenvolvimento de metodologia. 2a ed. Porto Alegre: Editora Rede Unida, 2018.

9. Instituto brasileiro de informação em ciência e tecnologia
Relatório de Progresso do Projeto Dimensionamento da Força de Trabalho em Secretarias de Saúde para a tomada de decisão em Gestão do Trabalho em Saúde a partir da coleta, estruturação e análise de dados quantitativos e qualitativos. Ref. FUNDEP 26656 - Ref. IBICT 0553/2018. [relatório técnico]. Brasília: IBCT, 2020.

10. Brasil. Decreto $n^{\circ} 7.508$, de 28 de junho de 2011. Regulamenta a lei n. 8080, de 19 de setembro de 1990, para dispor sobre a organização do Sistema Único de Saúde - SUS, o planejamento da saúde, a assistência à saúde e a articulação interfederativa, e dá outras providências. Diário Oficial da União. 29 jun. 2011.

11. Calvo MCM, Lacerda JT, Colussi CF, Schneider, IJC, Rocha TAH. Estratificação de municípios brasileiros para avaliação de desempenho em saúde. Epidemiol. Serv. Saúde [Internet]. 2016 dez. [acesso 2020 nov. 02]; 25 (4): 767-76. Disponivel em: http://www.scielo.br/scielo.php?script=sci_arttext\&pid=S2237-96222016000400767\&Ing=en.

12. Brasil. Ministério da Saúde. Conselho Nacional de Secretários de Saúde. Conselho Nacional de Secretarias Municipais de Saúde. Orientações Tripartite para o Planejamento Regional Integrado. Brasília: set. 2018.

13. Brasil. Ministério da Saúde, Comissão Intergestores Tripartite. Resolução n²3, de 17 de agosto de 2017. Estabelece diretrizes para os processos de Regionalização, Planejamento Regional Integrado, elaborado de forma ascendente, e Governança das Redes de Atenção à Saúde no âmbito do SUS. Diário Oficial da União, 18 ago. 2017.

14. Nascimento EPL, Carvalho DS, Carmona SAMLD, Barthmann VMC, organizadoras. Planejamento e dimensionamento da força de trabalho em saúde: material didático para secretarias de saúde. Brasília: IBICT, 2020.

15. Brasil. Ministério da Saúde. Portaria de Consolidação n 1 , de 28 de setembro de 2017. Consolidação das normas sobre os direitos e deveres dos usuários da saúde, a organização e o funcionamento do Sistema Único de Saúde. Diário Oficial da União. 03 out. 2017. 